ARTICLE

Received 31 Dec 2014 | Accepted 22 Jun 2015 | Published 29 Jul 2015

DOI: $10.1038 /$ ncomms 8874

OPEN

\title{
Deep and high-resolution three-dimensional tracking of single particles using nonlinear and multiplexed illumination
}

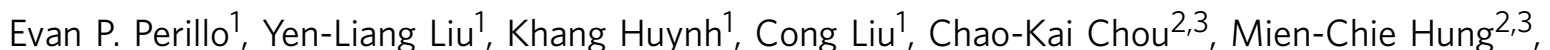
Hsin-Chih Yeh ${ }^{1} \&$ Andrew K. Dunn ${ }^{1}$

Molecular trafficking within cells, tissues and engineered three-dimensional multicellular models is critical to the understanding of the development and treatment of various diseases including cancer. However, current tracking methods are either confined to two dimensions or limited to an interrogation depth of $\sim 15 \mu \mathrm{m}$. Here we present a three-dimensional tracking method capable of quantifying rapid molecular transport dynamics in highly scattering environments at depths up to $200 \mu \mathrm{m}$. The system has a response time of $1 \mathrm{~ms}$ with a temporal resolution down to $50 \mu \mathrm{s}$ in high signal-to-noise conditions, and a spatial localization precision as good as $35 \mathrm{~nm}$. Built on spatiotemporally multiplexed two-photon excitation, this approach requires only one detector for three-dimensional particle tracking and allows for two-photon, multicolour imaging. Here we demonstrate three-dimensional tracking of epidermal growth factor receptor complexes at a depth of $\sim 100 \mu \mathrm{m}$ in tumour spheroids.

\footnotetext{
${ }^{1}$ Department of Biomedical Engineering, The University of Texas at Austin, 107 W Dean Keeton Street, C0800, Austin, Texas 78712, USA. ${ }^{2}$ Department of Molecular and Cellular Oncology, The University of Texas MD Anderson Cancer Center, 1515 Holocombe, Boulevard, Unit 108, Houston, Texas 77030-4009, USA. ${ }^{3}$ Center for Molecular Medicine and Graduate Institute of Cancer Biology, China Medical University, No. 91 Hsueh-Shih Road, Taichung 40402, Taiwan. Correspondence and requests for materials should be addressed to H.-C.Y. (email: tim.yeh@austin.utexas.edu) or to A.K.D. (email: adunn@mail.utexas.edu).
} 
S ingle-particle tracking (SPT) has enabled the direct observation of dynamic behaviours of particles (here a particle can be a single biomolecule, a molecular complex, a vesicle, a lipid granule or a viral capsid) inside complex biological systems $^{1-4}$, with particle localization precision better than the diffraction limit of light ${ }^{5,6}$. By trajectory analysis, SPT has provided insight into motor protein kinetics ${ }^{7,8}$, cellular membrane dynamics ${ }^{9-11}$, mRNA transport ${ }^{12,13}$ and virus internalization processes ${ }^{14,15}$. As the basis of passive microrheology, SPT has also shed light on the local environments of tracked particles through the observation of changes in particles' random movements ${ }^{16,17}$.

Whereas SPT is becoming a powerful research tool, all current techniques suffer from one or more of the following problems: shallow penetration depth (arising from the use of one-photon excitation ${ }^{18-20}$ ), limited $z$ tracking range (for example, total internal reflection fluorescence microscopy), poor temporal resolution (for example, frame-by-frame analysis in camera-based methods ${ }^{21,22}$ ) and low information content (for example, no information on the fluorescence lifetime ${ }^{23}$ ). As two-photon (2P) microscopy has become a standard method for deep tissue imaging ${ }^{24}$, a few reports demonstrated three-dimensional (3D) tracking based on 2P excitation. One of the earliest demonstrations of $2 \mathrm{P}$ tracking used an orbital scanning motion of the focused laser beam to track particles using a single detector ${ }^{5,25,26}$, but was limited to a temporal resolution of $20-32 \mathrm{~ms}$ due to mechanical scanning and signal demodulation. More recently, 3D tracking of gold nanorods with $2 \mathrm{P}$ excitation was demonstrated by exciting multiple foci and detecting fluorescence with an Electron Multiplying Charge Coupled Device (EMCCD) ${ }^{22}$, but the 3D temporal resolution was limited to $\sim 0.5 \mathrm{~s}$. Moreover, the use of a camera in multifocal $2 \mathrm{P}$ laser scanning microscopy limits the working depth of SPT in scattering samples ${ }^{27}$. Although SPT with superior temporal resolution (bounded mainly by the emission rate of the fluorescent label) and simultaneous fluorescence lifetime measurements have been achieved using confocal setups with 3-5 single-element/photon-counting detectors (photomultiplier tubes
(PMTs) or avalanche photodiodes) for spatial filtering ${ }^{18-20}$, these methods not only have limited working depth (not using $2 \mathrm{P}$ excitation for tracking) but also suffer from loss of signals due to the non-overlapping excitation and collection efficiency peaks in spatial filtering (Supplementary Fig. 3) ${ }^{28}$. Other confocal-based microscopes have been developed using only two detectors, which are capable of simultaneous spectroscopy measurements ${ }^{29}$; however, they still suffer from poor penetration depths in scattering samples such as tissues and multicellular structures, as well as low collection efficiency. Currently there is no single solution to all of the above issues.

To address this challenge, we have developed a 2P 3D SPT method capable of tracking particles at depths up to $200 \mu \mathrm{m}$ in scattering samples with $22 / 90[x y / z]-n m$ spatial localization precision and $1 \mathrm{~ms}$ response time. With bright fluorophores, we can achieve a temporal resolution down to $50 \mu \mathrm{s}$. At shallow depths, the localization precision can be as good as $35 \mathrm{~nm}$ in all 3D. The approach is based on passive pulse splitters used for nonlinear microscopy ${ }^{30}$ to achieve spatiotemporally multiplexed $2 \mathrm{P}$ excitation and temporally demultiplexed detection ${ }^{31}$ to discern the $3 \mathrm{D}$ position of the particle. The $\mathrm{z}$-tracking range is up to $\pm 50 \mu \mathrm{m}$ (limited by the objective z-piezo stage) and the method enables simultaneous fluorescence lifetime measurements on the tracked particles. Like some more recent techniques, this tracking method allows coupling trajectory data with traditional imaging to discern the local environment ${ }^{18,32}$. However, a major advantage of this method over confocal approaches is that it requires only one detector for SPT and is compatible with multicolour 2P microscopy. We describe our approach and demonstrate its capabilities by tracking single fluorescent beads in aqueous solutions that include scattering, as well as tracking prescribed motions in these controlled environments. We then demonstrate tracking of epidermal growth factor receptor (EGFR) complexes tagged with fluorescent beads in tumour spheroids, demonstrating deep 3D SPT in multicellular models. We have coined this technique tracking single particles using nonlinear and multiplexed illumination (TSUNAMI).
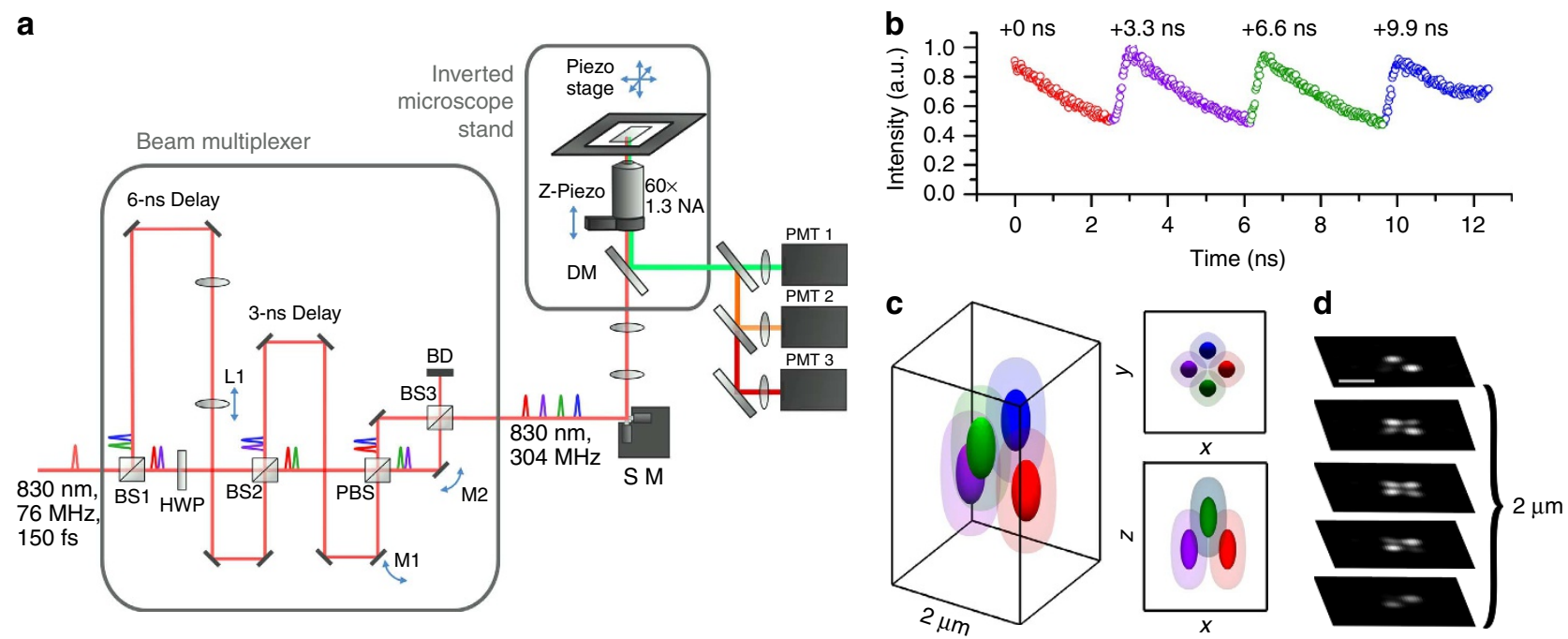

Figure 1 | 3D tracking instrumentation. (a) Schematic of the two-photon 3D tracking microscope. Spatiotemporal multiplexing is enabled through an optical system which utilizes two beam splitters (BS1 and BS2) to generate four beams, which can be quasi-independently controlled via mirrors (M1 and M2). Physical delay lines provide temporal separation. In this case, $6.6 \mathrm{~ns}(2 \mathrm{~m})$ and $3.3 \mathrm{~ns}(1 \mathrm{~m})$ path length delay lines create four beams with a period of $3.3 \mathrm{~ns}$ corresponding to an even division of the fundamental 13-ns period generated by the laser source (Mira 900, Coherent). Tracking actuation is performed using scanning mirrors (SM) and an objective focusing stage (z-piezo). (b) Photon-counting histogram of a particle centred in the middle of the four excitation focus demonstrating temporal offsets and power balance between the independent excitation beams. (c) An idealized image space projection of the tetrahedral PSF. (d) Experimental laser scanning image of a single $\phi 100-\mathrm{nm}$ fluorescent bead with simultaneous four beam excitation. Scale bar, $1 \mu \mathrm{m}$. HWP, half-wave plate; PBS, polarizing beam splitter; DM, dichroic mirror; BD, beam dump. 


\section{Results}

Spatiotemporal multiplexer design. In the spatiotemporal multiplexed scheme, laser pulses emitted with a 13-ns period from a Ti:sapphire oscillator are separated into four beams, which are delayed by $3.3 \mathrm{~ns}$ each and focused through a high numerical aperture objective at slightly offset $x y z$ positions. The four resulting $2 \mathrm{P}$ excitation volumes are arranged into a barely overlapped, tetrahedral geometry (Fig. 1c), to generate selective excitation equivalent to the spatial filtering condition in the previous four-detector confocal tracking set-up ${ }^{19}$, with each $2 \mathrm{P}$ excitation volume receiving laser pulses at a different time delay. For a fluorescent particle residing somewhere inside the excitation tetrahedron, its $2 \mathrm{P}$ emission is collected by a PMT (PMT1 in Fig. 1a). By time-correlated single-photon-counting (TCSPC) detection, each detected photon is assigned to a specific time gate $\left(\mathrm{G}_{1}-\mathrm{G}_{4}\right.$, here assuming the decay time is $\sim 4 \mathrm{~ns}$ or less) in the fluorescence decay histogram (Fig. 1b), and therefore attributed to an individual excitation volume. For a particle sitting at the centre of the tetrahedron, the resulting photon counts are approximately equal in all four time gates. An offset of the particle from the tetrahedron centre can be estimated from the normalized photon count differences in the four time gates (that is, error signals $E_{x}, E_{y}$ and $E_{z}$ in Supplementary Methods). Once the particle position offset is determined, a closed feedback loop then steers galvanometer mirrors and the objective z-piezo stage to lock the tracking beams on the particle. A particle's 3D trajectory is therefore determined directly from the controller output sent to the galvanometer and piezo actuators.

Spatiotemporal multiplexing has previously been explored for diffusion measurements ${ }^{33}$ and tracking ${ }^{34}$, but these methods rely on a picosecond pulsed laser for one-photon excitation and therefore are not suitable for use in multicellular models or tissues. Furthermore, only one photomultiplier tube (PMT1 in Fig. 1) is needed for SPT in our method, whereas 3-5 detectors are needed in confocal tracking set-ups ${ }^{18-20}$. In addition, the fluorescence lifetime of the tracked particle can be determined from the time-resolved photon data so long as the emitter lifetime is shorter than the gate width ( $<3.3 \mathrm{~ns}$; Supplementary Fig. 12) (ref. 26). As the laser beam is steered by active feedback to lock on the tracked particle, a large tracking range is achieved $( \pm 50 \mu \mathrm{m}$ in $z$ direction and $\pm 100 \mu \mathrm{m}$ in $x y$ direction) with minimal perturbation to the samples (whereas some confocal setups require the sample to be moved in order for $\mathrm{SPT}^{18,19}$ ).

Calibration and localization precision characterization. To validate our TSUNAMI microscope, we first tracked fluorescent beads ( $\phi 100 \mathrm{~nm}$, decay time $\sim 4.5 \mathrm{~ns}$ ) in aqueous solution (Fig. 2) and in $9 \%$ gelatin gel with $1 \%$ intralipid (a highly scattering environment; Supplementary Fig. 7). By following prescribed motions in these controlled environments ${ }^{5,35}$, we successfully characterized the localization precision, tracking speed limits, temporal resolution and tracking depth of our system.

From optical modelling, the optimal lateral and vertical separation distances between the $2 \mathrm{P}$ excitation volumes were estimated to be 500 and $1,000 \mathrm{~nm}$, respectively (Supplementary Fig. 3$)^{28}$. The alignment of the four excitation beams was verified by volumetric scanning with a fixed fluorescent bead (Fig. 1d). To determine particle localization uncertainty and maximum speed that our system can follow, we tracked a fixed fluorescent bead ( $\phi 100 \mathrm{~nm}, \mathrm{~F}-8803$, Life Technologies) loaded on an independent $x y z$ piezo stage $(\mathrm{P}-733 \mathrm{~K} 130, \mathrm{PI})^{5,35}$. The independent stage was programmed to move in a helical pattern (Fig. 2a). At an average speed of $2 \mu \mathrm{ms}^{-1}$, the estimated tracking errors (root mean squared) were $16.2 \mathrm{~nm}$ in $x, 16.7 \mathrm{~nm}$ in $y$ and $35.1 \mathrm{~nm}$ in $z$ (Supplementary Fig. 6). The localization precision stayed below
$45 \mathrm{~nm}$ when the particle speed was $<8 \mu \mathrm{ms}^{-1}$ (Fig. 2b). It should be noted that the fastest molecular motor known today ${ }^{36}$, FtsK, travels at $\sim 7 \mu \mathrm{ms}^{-1}$. Other than prescribed motions, we also tracked freely diffusing nanoparticles $(\phi 100 \mathrm{~nm}, \mathrm{~F}-8803$, Life Technologies) at various diffusion rates. Diffusion coefficients were estimated from fitting the mean-square displacement and compared with the values predicted from the Stokes-Einstein equation. Excellent agreements were seen in a wide range of diffusion coefficients $\left(0.07-4.3 \mu \mathrm{m}^{2} \mathrm{~s}^{-1}\right.$ in Fig. $2 \mathrm{c}$; Supplementary Fig. 5). Whereas a previous $3 \mathrm{D}$ SPT report has successfully tracked particles diffusing at $20 \mu \mathrm{m}^{2} \mathrm{~s}^{-1}$ (ref. 18), we note that diffusion coefficient of a free receptor complex on the cell membrane is roughly on the order of $0.02 \mu \mathrm{m}^{2} \mathrm{~s}^{-1}$ (ref. 37), and the fast diffusion coefficient of proteins in the cytosol is on the order of $5 \mu \mathrm{m}^{2} \mathrm{~s}^{-1}$ (ref. 38). Our system thus has no problem to probe rapid molecular transport dynamics inside cells.

Temporal resolution characterization. Temporal resolution of our tracking system is defined as how fast the particle position is discerned in $3 \mathrm{D}$ space with reasonable localization accuracy. Although our control loop period is $1-5 \mathrm{~ms}$, we emphasize that our temporal resolution can be significantly better than $1 \mathrm{~ms}$ by outputting the individual photon event data (Time Tag) from the TCSPC board (while the control loop period remains at 1-5 ms). In offline analysis, trajectories can be resampled with temporal resolution down to $50 \mu$ s if the particle has a sufficiently high brightness (Supplementary Fig. 11). In this condition, the trajectory is plotted from a combination of the original control loop rate voltage outputs and $n$-samples of higher time resolution localizations relative to the current beam position. Localizations are performed using rebinned histograms with photons arriving only within the super-sampled time period.

Tracking depth characterization. To mimic $3 \mathrm{D}$ tracking in a turbid tissue sample, $\phi 40 \mathrm{~nm}$ fluorescent beads were fixed within a $9 \%$ gelatin gel with $1 \%$ intralipid (Supplementary Fig. 7). When tracking beads undergoing prescribed motion at $\sim 10-\mu \mathrm{m}$ depth, localization uncertainty stayed the same in $x y$ direction but slightly increased in $z$ direction $(60 \mathrm{~nm})$. Localization uncertainty further increased to $\sim 89 \mathrm{~nm}$ at the depths of 100 through $200 \mu \mathrm{m}$, while localization uncertainty stayed below $22 \mathrm{~nm}$ in $x y$ direction at both depths. This reduction in $z$ localization precision may be due to an elongated molecular detection function (Supplementary Fig. 4) that occurs when light is focused through scattering samples, which may blur the beams along the $z$ dimension ${ }^{39}$ and lower the optical contrast signal required to lock onto the target. Despite this slight reduction in $z$ tracking accuracy at depth, TSUNAMI is capable of maintaining better than 100-nm axial localization through $200 \mu \mathrm{m}$ of a scattering sample.

2P-3D-SPT in monolayer cultures and cancer spheroids. Here we used the endocytosis and subcellular trafficking of EGFR complexes in A431 monolayer culture and tumour spheroids as a model system for instrument validation. We tracked singlenanoparticle ( $\phi 40 \mathrm{~nm}$, F8770, Life Technologies)-bound EGFR complexes (see Supplementary Online Methods) in monolayer cell cultures (Fig. 3a) and $\sim \phi 100-\mu \mathrm{m}$ spheroids (Fig. $4 \mathrm{a}$ ) for periods up to $10 \mathrm{~min}$. Before SPT, $2 \mathrm{P}$ fluorescence images were taken of the surrounding cellular environment. Staining of the plasma membrane and nucleus allows co-registration of the particle trajectory with cellular landmarks. In post processing, the trajectory and cellular images are co-registered (Fig. 3b) to visualize EGFR entry pathways.

We found that out of 100 trajectories $\sim 80 \%$ of EGFRs had been internalized into the cells within $\sim 6 \mathrm{~min}$. We notice 
a

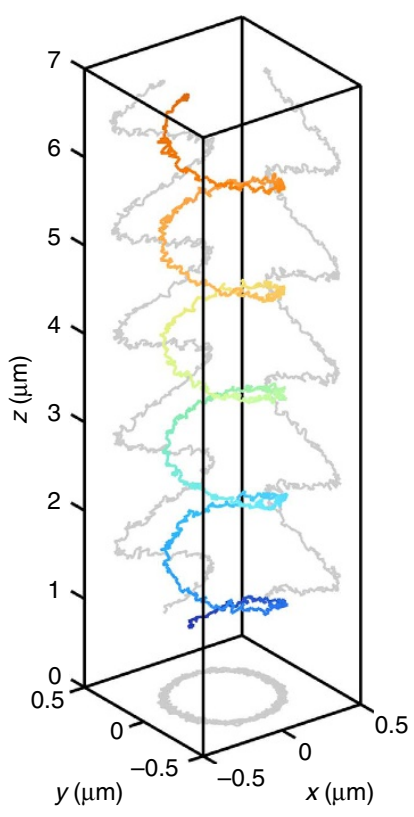

b

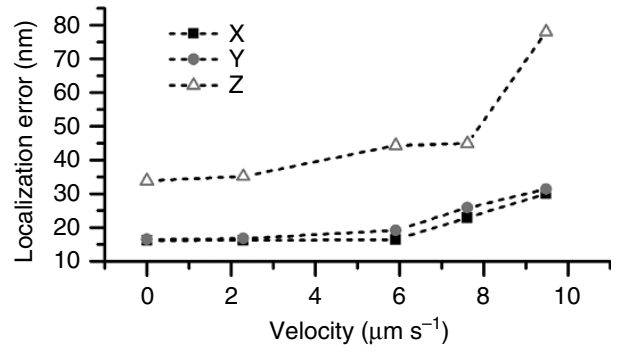

C

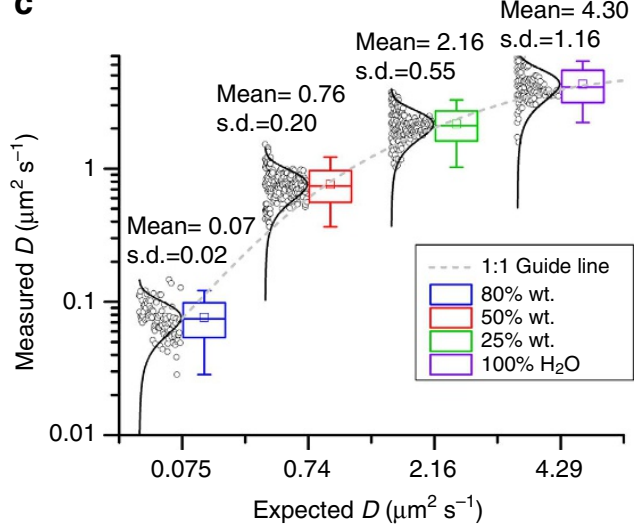

d

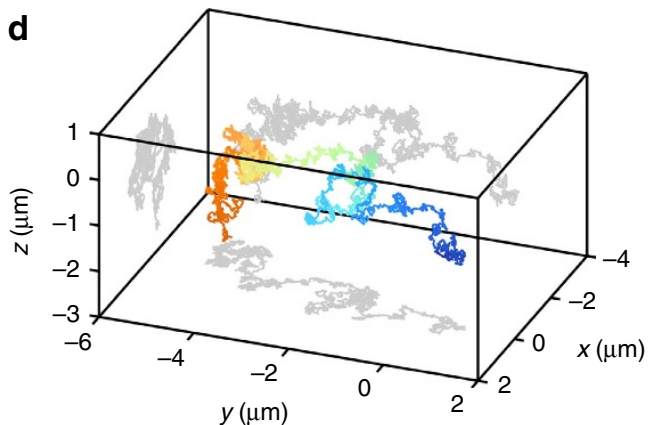

e

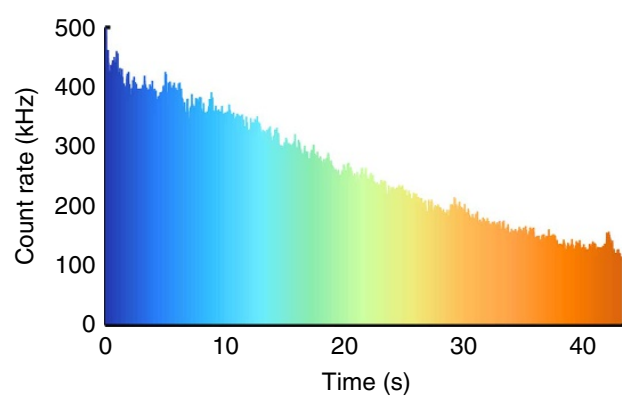

Figure 2. | Characterization of directed motion and free diffusion. (a) Example helical trajectory of a $\phi 100$-nm fluorescent bead moved through a known path using an independent 3D piezo stage (P-733K130, PI). The path duration was $7 \mathrm{~s}$ with an average velocity of $2.1 \mu \mathrm{ms} \mathrm{s}^{-1}$. The tracking system reproduced the true trajectory accurately with $16.5 \mathrm{~nm}$ uncertainty in $x$ and $y$ and $33.3 \mathrm{~nm}$ in $z$. Rainbow coloration corresponds to time with blue representing the beginning of the trajectory. (b) Several helical trajectories were performed with varying mean velocities. The particle localization uncertainty increases with increasing velocities up to $10 \mu \mathrm{ms}^{-1}$, where the particle speed is too fast for the controller to target lock. (c) Box plot with histograms of measured versus theoretical diffusion coefficients, D, for $80 \%$ wt. glycerol, $50 \%$ wt. glycerol, $20 \%$ wt. glycerol and $100 \%$ water, respectively. The measured central tendency of the diffusion coefficients were found to agree with the Stokes-Einstein equation for wide range of values $\left(0.07-4.3 \mu \mathrm{m}^{2} \mathrm{~s}^{-1}\right)$. The agreement with theory can been seen by how closely the data follow the 1:1 guide line (plotted on semilog scale). (d) An example trajectory of a $\phi 100-\mathrm{nm}$ fluorescence bead in an $80 \% \mathrm{wt}$. glycerol solution, free diffusion was observed over $43 \mathrm{~s}$. (e) Particle photon count rate versus time, a monotonically decreasing count rate indicates that a single bead, or aggregate, is stably locked in the field of view for the entire duration of the trajectory with no other particles entering or leaving the field of view.

transport modes similar to those described in prior work ${ }^{40}$, (Fig. 3d). The average velocity $\left(2 \mu \mathrm{ms}^{-1}\right)$ and total transport length $(1-2 \mu \mathrm{m})$ during internalization are in good agreement with values previously reported.

For spheroid models, we measured EGFR entry pathways at a variety of depths from 20 to $100 \mu \mathrm{m}$ past the coverslip (Fig. 4c; Supplementary Figs 13 and 14) and found good agreement in terms of the speed and transport length during internalization (Fig. 4d). Although the required power to obtain clean spheroid images expectedly increased with imaging depth, the total signal count rate $(500-800 \mathrm{kHz})$ and signal to noise remained well within a threshold required for target locking. EGFR trajectories were easily measured at a depth of $100 \mu \mathrm{m}$ for up to $10 \mathrm{~min}$ with minimal photobleaching. To evaluate whether the trajectories are representative of biological events or system artifacts, we measured trajectories under a control environment with an endocytosis inhibitor, sodium azide and low temperature $\left(<20^{\circ} \mathrm{C}\right)$ (Supplementary Fig. 15). Of the 30 inhibited trajectories, we observed no high-speed transport modes and only external membrane-bound slow diffusion with an average velocity of $\sim 0.2 \mu \mathrm{ms}^{-1}$. The average tracking duration was $500 \mathrm{~s}$.

Clearly this technique is capable of measuring biologically relevant activity in the high-background environment of monolayer and spheroid models with instantaneous transport speeds up to $7 \mu \mathrm{ms}^{-1}$. We emphasize that TSUNAMI is capable of measuring EGFR translocation pathways at depths $10 \times$ what was previously possible through highly scattering, cell dense samples. In addition, the multicolour, multiresolution $2 \mathrm{P}$ fluorescence imaging functionality is inherently integrated to allow for co-registration of deep trajectories to local cellular/tissue environment in $3 \mathrm{D}$.

\section{Discussion}

Whereas a few reports demonstrated 3D SPT in monolayer cell cultures using $2 \mathrm{P}$ excitation ${ }^{5,22}$, to the best of our knowledge high-resolution subsurface particle tracking in 3D has never been demonstrated in highly scattering multicellular environments. One recent report demonstrates deep 3D SPT light sheet microscopy using astigmatism ${ }^{41}$; however, the capability is limited by the use of an EMCCD (16-ms temporal resolution), and would prove difficult to implement multicolour tracking or simultaneous lifetime measurement schemes. Furthermore, we expect an astigmatic point spread function (PSF) would have significantly decreased localization precision when tracking at depth (greater than $10 \mu \mathrm{m})^{21}$.

Other than penetration depth, $2 \mathrm{P}$ excitation allows us to excite multiple fluorophores simultaneously, which greatly facilitates multicolour detection (Fig. 3) ${ }^{42}$. The two factors that determine the fundamental limit of our temporal resolution are the detector and the tracer. The timing resolution (full-width at half- 
a

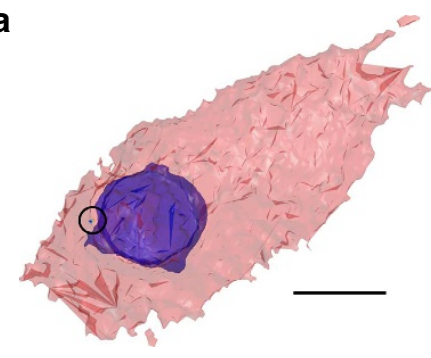

b

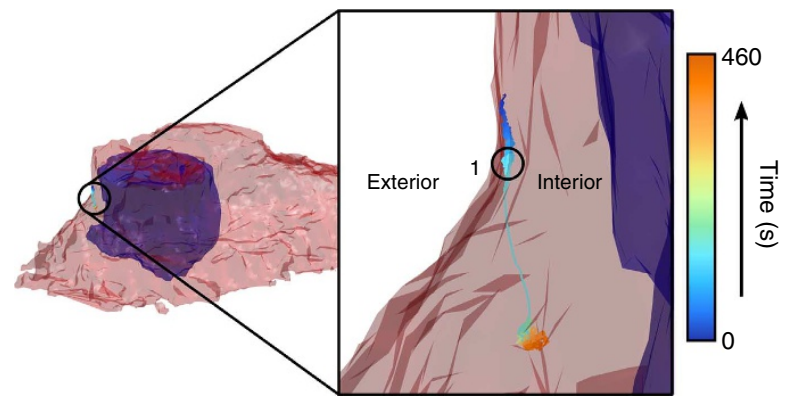

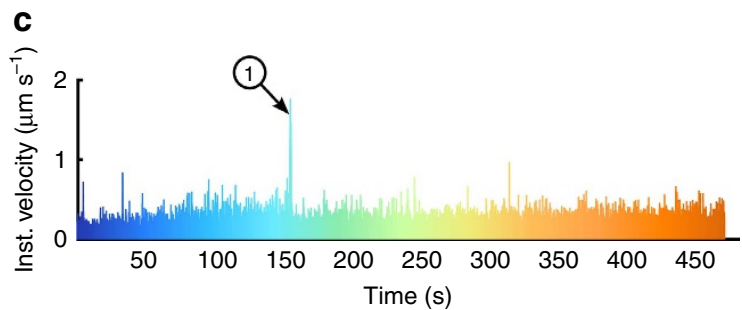

d

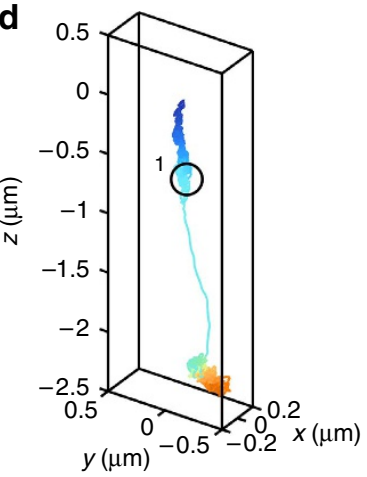

Figure 3 | 3D single-particle tracking in monolayer cell cultures. The capability to track singly labelled EGF molecules is demonstrated in a live-cell environment. (a) 3D isocontour model of the cell structure with staining for plasma membrane (red) and nuclei (blue). Scale bar, $10 \mu \mathrm{m}$. (b) Cell isocontour model plotted with trajectory overlaid (inset: zoomed view of the particle trajectory, a single fluorescent-labelled EGF molecule was tracked for a duration of 460 s with rainbow coloration corresponding to time, where blue marks the beginning). (c) Instantaneous velocity graph with corresponding colour scheme. For the first $150 \mathrm{~s}$, the particle undergoes slow directed diffusion (mean velocity $\sim 0.4 \mu \mathrm{ms}^{-1}$ ) along the exterior of the cell. A ramp in velocity is observed followed by a period of high average velocity (start denoted as point 1 ), $\sim 2 \mu \mathrm{ms}^{-1}$, and unidirectional transport of $1 \mu \mathrm{m}$. This behaviour indicates some form of internalization into the cell or transport within the cell. (d) Trajectory plotted with no cell contour overlay.

maximum of the instrument response function) of our detector (H7422P-40, Hamamatsu) is about 230 ps (Supplementary Fig. 12). Whereas this response time is typical in photoncounting detectors, it is still orders of magnitude faster than charge-coupled devices. It is well known that localization accuracy of a fluorescent particle relies heavily on the brightness and photostability of this fluorescent molecule ${ }^{43}$. Assuming that a bright fluorophore has an emission rate of $10^{8} \mathrm{~s}^{-1}$ and $10 \%$ of emitted photons are collected, the detected photons could exceed $10^{7}$ per second. As $\sim 100$ photons are needed for particle position determination with moderate precision, this could mean that the ultimate temporal resolution for SPT is $\sim 10 \mu \mathrm{s}$. Recently, another group reported achieving $10 \mathrm{~nm} 3 \mathrm{D}$ localization precision and $10-\mu$ s temporal resolution in confocal 3D tracking of a giant quantum dot (QD) cluster $(\sim 40$ QDs $)^{18}$. As our 3D tracking approach has better collection efficiency (non-descanned detection and the emission light is not split among multiple detectors), we see no potential problem for our system to reach similar temporal resolution and localization precision using a tracer system with ultrahigh emission rate but with a short lifetime.

Despite a shorter time gate of $3.3 \mathrm{~ns}$, demultiplexing of longer lifetime emitters can be performed by applying a fluorescence correction factor in our tracking algorithm (further discussed in Supplementary Fig. 12). The correction factor allows tracking of lifetimes up to $4.5 \mathrm{~ns}$ by subtracting the estimated crosstalk from the previous time gate. The correction factor must be adjusted for fluorophores of different lifetimes. With a correspondingly smaller correction factor, we demonstrate tracking a short lifetime emitter, Cy5-tagged beads $(1.18 \mathrm{~ns})$, and show that lifetime can be accurately measured ( $\pm 0.08 \mathrm{~ns}$ ) during tracking (Supplementary Fig. 12). However, for extremely long lifetime emitters, such as quantum dots, or emitters whose lifetime changes dynamically, a lower repetition rate source or a pulse picker would be needed, in conjunction with longer physical delay lines.

Moving the beam through the sample instead of moving the sample itself ${ }^{18,19,26}$ has the additional advantage that beam steering is generally faster than the movement of a potentially heavy stage. Also, steering the beam is particularly suitable for applications in neuroscience, such as patching clamping, which requires stationary samples. While the stage response frequencies ( $10 \mathrm{kHz}$ for the galvo mirrors and $300 \mathrm{~Hz}$ for the objective $\mathrm{z}$-piezo stage) do not determine the temporal resolution of our tracking system, they impose an upper limit on the observable particle speeds ${ }^{35}$. The way to bypass this limitation in mechanical scanning and completely rule out the possibility that the observed dynamics are influenced by the objective motion (via mechanical coupling through the immersion medium) is to use fast adaptive optical elements in both lateral $\operatorname{scan}^{26,44}$ and axial focusing ${ }^{45}$.

An important characteristic of our tracking microscope lies in the fact that it is readily compatible with a number of fluorescence spectroscopy or microscopy techniques for probing molecular interactions, potentially at the true single-molecule level ${ }^{46}$, including fluorescence resonance energy transfer ${ }^{47}$ (via lifetime measurements), multicolour single-molecule imaging/ spatiotemporal colocalization analysis ${ }^{48}$, step counting ${ }^{49,50}$, and mean-square displacement analysis ${ }^{44}$. Since only one detector is used for 3D tracking, additional detectors can be easily added and used for simultaneous, multicolour detection, which can provide a structural overview of the particle's surrounding environment or indicate molecular interactions. On the other hand, it is not straightforward to detect the second colour simultaneously on the traditional confocal tracking set-ups that employ 3-5 detectors for spatial filtering ${ }^{18-20}$, as twice as many detectors and serious alignment effort may be required for multicolour detection. The unique nature of the tetrahedral point spread function allows 
a

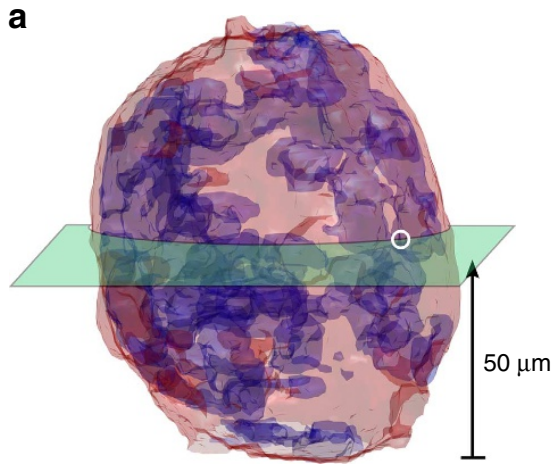

C

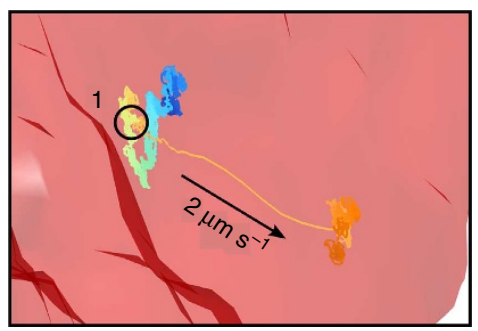

b

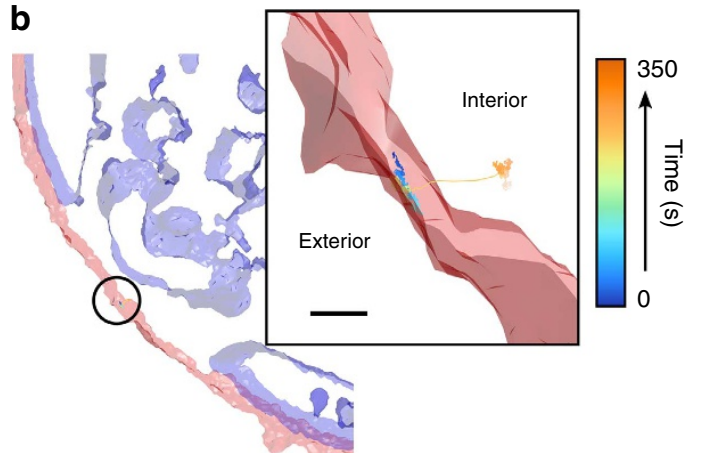

d

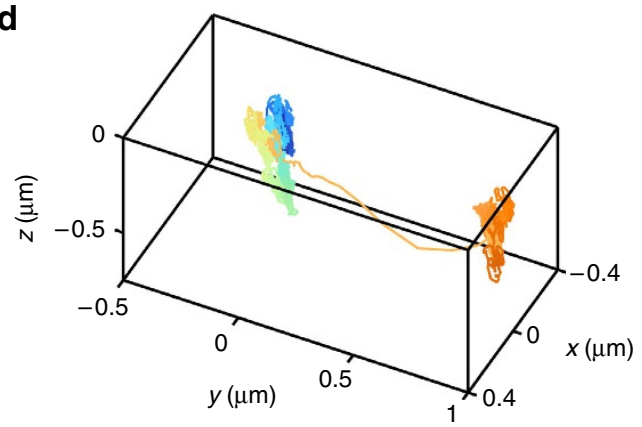

e

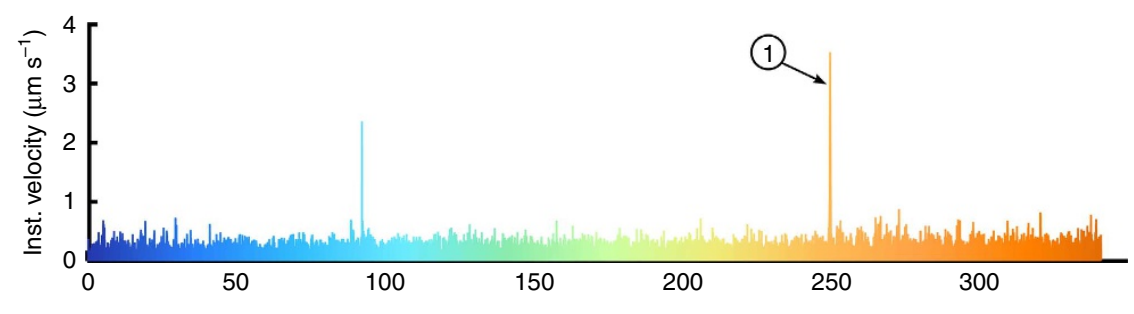

Figure 4. I Deep single-particle tracking of EGFR in spheroid model. Demonstration of deep 3D SPT in a spheroid model. (a) 3D isocontour of a $\phi 100-\mu \mathrm{m}$ spheroid taken with $2 \mathrm{P}$ laser scanning microscopy staining for the plasma membrane (red) and nuceli (blue). The highlighted slice denotes the $z$ plane (at $50-\mu \mathrm{m}$ depth) where the trajectory was measured, with the white circle marking the location of the trajectory on the spheroid. (b) Isocontour model of the \pm 5 - $\mu \mathrm{m}$ slice taken $50-\mu \mathrm{m}$ deep within the spheroid. Plasma membrane and nuclei are overlaid with the trajectory (black circle), (inset: zoomed view of the trajectory). (c) Zoomed view of the trajectory. The trajectory begins inside the cell with slow displacement (mean velocity $\sim 0.17 \mu \mathrm{ms} \mathrm{s}^{-1}$ ) for $250 \mathrm{~s}$. Point 1 (black circle) is where velocity increases to $2 \mu \mathrm{m} \mathrm{s}^{-1}$ and is sustained for $0.5 \mathrm{~s}$ in a unidirectional manner. (d) Trajectory plotted without cell overlay, the total transport length within the high velocity region is $1 \mu \mathrm{m}$. (e) Instantaneous velocity plot over the duration of the trajectory. Scale bar, $600 \mathrm{~nm}$.

easily configurable beam spacing which may be optimized for measurements such as $3 \mathrm{D}$ pair correlation analysis $5 \mathrm{f}$.

Here we demonstrate a new 2P 3D SPT microscope (TSUNAMI) that addresses fundamental limitation of deep and high-resolution SPT in the 3D space. Extended from our current tracking results in multicellular models, we are working towards direct in vivo 3D SPT at high spatiotemporal resolution. Our system will allow researchers to explore new questions in receptor transport and dynamic processes directly in $3 \mathrm{D}$ tissues.

\section{Methods}

2P-3D-SPT instrumentation. The spatiotemporal beam multiplexer (Fig. 1) is created entirely from passive optics comprises beam splitters, mirrors and waveplates (Supplementary Fig. 1). A single-pulse train from a modelocked $\mathrm{Ti}: \mathrm{Al}_{2} \mathrm{O}_{3}$ laser (Mira 900, Coherent) tuned to $835 \mathrm{~nm}$ is used as the primary beam, which then gets multiplexed into four beams offset in time and space. For temporal offsets, it was determined that to equally space four beams with an original $76 \mathrm{MHz}$ repetition rate the delay time must be $3.3 \mathrm{~ns}$, which corresponds to $\sim 1 \mathrm{~m}$ physical path length. For spatial offsets, each beam is first coaligned onto the primary optical axis. Adjusters that control $x$ and $y$ offsets are moved until a lateral spacing of $\sim 500 \mathrm{~nm}$ is achieved in the image plane. Alignment repeatability is verified by projecting fiduciary marks onto the laser scanning microscopy image during alignment such that each beam's centre is aligned to the same point in space to sub-100 nm repeatability. Axial spacing is controlled using a telescope assembly placed in the optical path of one beam pair to adjust their collimation. The alignment of axial spacing is verified using molecular detection function 3D scans (Supplementary Fig. 4) until a spacing of $1 \mu \mathrm{m}$ is achieved (Supplementary Fig. 3). Following the optical multiplexer the beams pass through a galvo scanning system $(6125 \mathrm{H}$, Cambridge Technology), before being focused through a $60 \times 1.3$ numerical aperture silicone oil objective (UPLSAPO60X, Olympus).

For a typical experiment, we use an average laser power of $\sim 2 \mathrm{~mW}$ per beam $(8 \mathrm{~mW})$ total at the objective back aperture. For a typical $\phi 40 \mathrm{~nm}$ fluorescent bead (F8770, Life Technologies.) photon count rates are in the range of $500-800 \mathrm{kHz}$ (Supplementary Fig. 17) and targets can be tracked for durations up to $10 \mathrm{~min}$ (Supplementary Fig. 16). Background fluorescence signal is on the order of $3 \mathrm{kHz}$ that includes a $150 \mathrm{~Hz}$ background signal from the detector. Signal-to-noise ratios are typically above 20 .

Demultiplexing emission signal. Demultiplexing of fluorescence emission is performed by time resolving the excitation contributions of each of the four multiplexed beams with fast photon-counting electronics. The fluorescence signal detected is an interwoven stream of photons excited by all four excitation beams. That signal can be time gated with $3.3 \mathrm{~ns}$ increments to effectively isolate the signal contributions from each beam and consequently isolate the signal contribution in space as well. Emission demultiplexing is performed electronically via TCSPC analysis. Fluorescence signals are detected by a cooled GaAsP photomultiplier tube with 5-mm square active area (H7422PA-40, Hamamatsu) in non-descanned configuration. The current output from the PMT is amplified through a $2 \mathrm{GHz}$ cutoff bandwidth preamplifier (HFAC-26, Becker and Hickl GmbH) and sent into a photon-counting board (SPC-150, Becker and Hickl $\mathrm{GmbH}$ ) to be counted and 
correlated to the $76-\mathrm{MHz}$ reference clock of the laser oscillator. Given the electronics setup our fundamental timing resolution is on the order of the instrument response frequency, which was measured to be 230 ps full-width at half-maximum (Supplementary Fig. 12).

Tracking control software. The tracking acquisition control loop is run entirely in LabVIEW (National Instruments) on the Windows 7 operating system. Time-resolved signals from the TCSPC board can be read into LabVIEW by two methods: (1) histogram mode and (2) First In, First out (FIFO) mode (also known as Time Tag). In histogram mode, the TCSPC board performs on board histogramming of the photons detected during a single-control loop period (5 ms) and sends the data to LabVIEW for processing into new control signals (Supplementary Fig. 2). In FIFO mode, each photon event is recorded by the board and processed on the fly in the LabVIEW control loop. For a loop rate of $5 \mathrm{~ms}$ and typical particle count rate of $500 \mathrm{kHz}$, this requires time binning 2,500 photons that are represented with 12-bit precision. This task is easily achievable with current computing hardware. The data on each photon event can be recorded for post processing and re-binning to achieve timing resolutions down to $50 \mu$ s with sufficiently bright particles (Supplementary Figs 11 and 18). Deterministic timing for the LabVIEW control loop is achieved by forcing the program to run on a hardware-timed clock from a peripheral component interface (PCI) data acquisition board (PCIe-6353, National Instruments). Using this hardware-timed loop method periods down to $1 \mathrm{~ms}$ can be requested with no missed cycles for up to $60 \mathrm{~s}$. At $5 \mathrm{~ms}$, the timed loop can run indefinitely with no missed cycles up to $20 \mathrm{~min}$.

Control signals are generated by taking the ratio of the summed photon counts in each of the four time gates. Following the below formulas,

$$
\begin{gathered}
E_{x}=\frac{G_{2}-G_{1}}{G_{2}+G_{1}} \\
E_{y}=\frac{G_{4}-G_{3}}{G_{4}+G_{3}} \\
E_{z}=\frac{\left(G_{4}+G_{3}\right)-\left(G_{2}+G_{1}\right)}{\left(G_{1}+G_{2}+G_{3}+G_{4}\right)}
\end{gathered}
$$

where $G_{1}, G_{2}, G_{3}$ and $G_{4}$ are the total photon counts in each time gate. Error signals $E_{x}, E_{y}$ and $E_{z}$ are modified by a proportional controller before being sent out by the PCIe- 6353 board as analogue signals to their respective actuators (galvos for $x$ and $y$, and objective piezo stage (P-726 PIFOC, PI) for $z$ ).

Sample preparation. EGFR-overexpressed A431 skin cancer cell was purchased from American Type Culture Collection and cultured in Dulbecco's Modified Eagle Medium (Cat. No. 11995-065, Life Technologies) supplemented with 5\% fetal bovine serum (Cat. No. SH30071, Thermo Scientific). The cell cultures were kept in humidified atmosphere with $5 \% \mathrm{CO}_{2}$ in air at $37^{\circ} \mathrm{C}$. Agarose-coated 96 -well plates were used to cultivate A431 spheroids. The spheroids were prepared as previously described ${ }^{52}$ and the plates were incubated for $96 \mathrm{~h}$ in a humidified atmosphere with $5 \% \mathrm{CO}_{2}$ at $37^{\circ} \mathrm{C}$. Cell-seeding density was 125 cells per well (Supplementary Fig. 10). Both monolayer cells and spheroids were kept for additional $24 \mathrm{~h}$ under serum-starvation condition before EGFR tracking. Plasma membrane was stained with CellMask and surface EGFRs were labelled with fluorescent nanoparticles (Supplementary Fig. 8). To label EGFRs, the cells were incubated with $1.5 \%$ bovine serum albumin solution (Cat. No. S7806) for $15 \mathrm{~min}$ at $37^{\circ} \mathrm{C}$, and then EGFRs were labelled with biotinylated anti-EGFR antibodies $\left(200 \mathrm{ng} \mathrm{ml}^{-1}\right.$ in $1.5 \%$ bovine serum albumin solution; EGFR Ab-3, Cat. No. MS-311-B, Thermo Scientific) for $15 \mathrm{~min}$ at $37^{\circ} \mathrm{C}$ (Supplementary Fig. 9). The antibody solution was removed and cells were washed twice using PBS. The stock solution of $\phi 40-\mathrm{nm}$ fluorescent nanoparticles (FluoSpheres NeutrAvidin-Labelled Microspheres No. F8770, Life Technologies) was sonicated for $10 \mathrm{~min}$ and then diluted to $100 \mathrm{pM}$ in DMEM. This solution was added into samples for $5 \mathrm{~min}$ at $37^{\circ} \mathrm{C}$.

\section{References}

1. Saxton, M. J. \& Jacobson, K. Single-particle tracking: applications to membrane dynamics. Annu. Rev. Biophys. Biomol. Struct. 26, 399 (1997).

2. Fakhri, N. et al. High-resolution mapping of intracellular fluctuations using carbon nanotubes. Science 344, 1031-1035 (2014).

3. Lowe, A. R. et al. Selectivity mechanism of the nuclear pore complex characterized by single cargo tracking. Nature 467, 600-603 (2010).

4. Kusumi, A., Tsunoyama, T. A, Hirosawa, K. M., Kasai, R. S. \& Fujiwara, T. K. Tracking single molecules at work in living cells. Nat. Chem. Biol. 10, 524-532 (2014).

5. Levi, V., Ruan, Q. \& Gratton, E. 3-D particle tracking in a two-photon microscope: application to the study of molecular dynamics in cells. Biophys. J. 88, 2919-2928 (2005).

6. Cang, H., Xu, C. S., Montiel, D. \& Yang, H. Guiding a confocal microscope by single fluorescent nanoparticles. Opt. Lett. 32, 2729-2731 (2007).

7. Yildiz, A. et al. Myosin V walks hand-over-hand: single fluorophore imaging with 1.5-nm localization. Science 300, 2061-2065 (2003)
8. Gelles, J., Schnapp, B. \& Sheetz, M. Tracking kinesin-driven movements with nanometre-scale precision. Nature 331, 450-453 (1988).

9. Fujiwara, T., Ritchie, K., Murakoshi, H., Jacobson, K. \& Kusumi, A. Phospholipids undergo hop diffusion in compartmentalized cell membrane. J. Cell Biol. 157, 1071-1081 (2002).

10. Dahan, M. et al. Diffusion dynamics of glycine receptors revealed by single-quantum dot tracking. Science 302, 442-445 (2003).

11. Andrews, N. L. et al. Actin restricts FceRI diffusion and facilitates antigen-induced receptor immobilization. Nat. Cell Biol. 10, 955-963 (2008).

12. Park, H. Y. et al. Visualization of dynamics of single endogenous mRNA labeled in live mouse. Science 343, 422-424 (2014)

13. Shav-Tal, Y. et al. Dynamics of single mRNPs in nuclei of living cells. Science 304, 1797-1800 (2004)

14. Huang, B., Jones, S. A., Brandenburg, B. \& Zhuang, X. Whole-cell 3D STORM reveals interactions between cellular structures with nanometer-scale resolution. Nat. Methods 5, 1047-1052 (2008).

15. Arhel, N. et al. Quantitative four-dimensional tracking of cytoplasmic and nuclear HIV-1 complexes. Nat. Methods 3, 817-824 (2006).

16. Mason, T. G. \& Weitz, D. A. Optical Measurements of Frequency-Dependent Linear Viscoelastic Moduli of Complex Fluids. Phys. Rev. Lett. 74, 1250-1253 (1995).

17. Wirtz, D. Particle-tracking microrheology of living cells: principles and applications. Annu. Rev. Biophys 38, 301-326 (2009).

18. Welsher, K. \& Yang, H. Multi-resolution 3D visualization of the early stages of cellular uptake of peptide-coated nanoparticles. Nat. Nanotechnol. 9, 198-203 (2014).

19. Wells, N. P. et al. Time-resolved three-dimensional molecular tracking in live cells. Nano Lett. 10, 4732-4737 (2010).

20. Sahl, S. J., Leutenegger, M., Hilbert, M., Hell, S. W. \& Eggeling, C. Fast molecular tracking maps nanoscale dynamics of plasma membrane lipids. Proc Natl. Acad. Sci. USA 107, 6829-6834 (2010).

21. Thompson, M. a, Lew, M. D., Badieirostami, M. \& Moerner, W. E. Localizing and tracking single nanoscale emitters in three dimensions with high spatiotemporal resolution using a double-helix point spread function. Nano Lett. 10, 211-218 (2010).

22. Van den Broek, B., Ashcroft, B., Oosterkamp, T. H. \& van Noort, J. Parallel nanometric 3D tracking of intracellular gold nanorods using multifocal two-photon microscopy. Nano Lett. 13, 980-986 (2013).

23. Cang, H., Shan, Xu, C. \& Yang, H. Progress in single-molecule tracking spectroscopy. Chem. Phys. Lett. 457, 285-291 (2008).

24. Helmchen, F. \& Denk, W. Deep tissue two-photon microscopy. Nat. Methods 2, 932-940 (2005)

25. Kis-Petikova, K. \& Gratton, E. Distance measurement by circular scanning of the excitation beam in the two-photon microscope. Microsc. Res. Tech. 63, 34-49 (2004)

26. McHale, K., Berglund, A. J. \& Mabuchi, H. Quantum dot photon statistics measured by three-dimensional particle tracking. Nano Lett. 7, 3535-3539 (2007).

27. Kim, K. H. et al. Multifocal multiphoton microscopy based on multianode photomultiplier tubes. Opt. Express 15, 11658-11678 (2007).

28. Liu, C. et al. 3D single-molecule tracking using one- and two-photon excitation microscopy. SPIE Proc. 8950, 1-9 (2014)

29. Cang, H., Wong, C. M., Xu, C. S., Rizvi, A. H. \& Yang, H. Confocal three dimensional tracking of a single nanoparticle with concurrent spectroscopic readouts. Appl. Phys. Lett. 88, 223901 (2006).

30. Ji, N., Magee, J. \& Betzig, E. High-speed, low-photodamage nonlinear imaging using passive pulse splitters. Nat. Methods 5, 197-202 (2008).

31. Cheng, A., Gonçalves, J. T., Golshani, P., Arisaka, K. \& Portera-Cailliau, C Simultaneous two-photon calcium imaging at different depths with spatiotemporal multiplexing. Nat. Methods 8, 139-142 (2011).

32. Katayama, Y. et al. Real-time nanomicroscopy via three-dimensional single-particle tracking. Chemphyschem 10, 2458-2464 (2010)

33. Dertinger, T. et al. Two-focus fluorescence correlation spectroscopy: a new too for accurate and absolute diffusion measurements. Chemphyschem 8, 433-443 (2007).

34. Germann, J. A. \& Davis, L. M. Three-dimensional tracking of a single fluorescent nanoparticle using four-focus excitation in a confocal microscope. Opt. Express 22, 9830-9834 (2014).

35. Juette, M. F. \& Bewersdorf, J. Three-dimensional tracking of single fluorescent particles with submillisecond temporal resolution. Nano Lett. 10, 4657-4663 (2010).

36. Lee, J. Y., Finkelstein, I. J., Crozat, E., Sherratt, D. J. \& Greene, E. C. Single-molecule imaging of DNA curtains reveals mechanisms of KOPS sequence targeting by the DNA translocase FtsK. Proc. Natl Acad. Sci. USA 109, 6531-6536 (2012).

37. Pyenta, P. S., Schwille, P., Webb, W. W., Holowka, D. \& Baird, B. Latera diffusion of membrane lipid-anchored probes before and after aggregation of cell surface IgE-receptors. J. Phys. Chem. A 107, 8310-8318 (2003). 
38. Griffin, E., Odde, D. \& Seydoux, G. Regulation of the MEX-5 gradient by a spatially segregated kinase/phosphatase cycle. Cell 146, 955-968 (2011).

39. Hayakawa, C., Venugopalan, V., Krishnamachari, V. \& Potma, E. Amplitude and phase of tightly focused laser beams in turbid media. Phys. Rev. Lett. 103, 043903 (2009).

40. De Bruin, K. et al. Cellular dynamics of EGF receptor-targeted synthetic viruses. Mol. Ther. 15, 1297-1305 (2007).

41. Spille, J.-H., Kaminski, T., Königshoven, H.-P. \& Kubitscheck, U. Dynamic three-dimensional tracking of single fluorescent nanoparticles deep inside living tissue. Opt. Express 20, 19697-19707 (2012).

42. Heinze, K. G., Koltermann, A. \& Schwille, P. Simultaneous two-photon excitation of distinct labels for dual-color fluorescence crosscorrelation analysis. Proc. Natl Acad. Sci. USA 97, 10377-10382 (2000).

43. Thompson, R. E., Larson, D. R. \& Webb, W. W. Precise nanometer localization analysis for individual fluorescent probes. Biophys. J. 82, 2775-2783 (2002).

44. Wang, Q. \& Moerner, W. E. Single-molecule motions enable direct visualization of biomolecular interactions in solution. Nat. Methods 11, 555-558 (2014).

45. Juette, M. F., Rivera-Molina, F. E., Toomre, D. K. \& Bewersdorf, J. Adaptive optics enables three-dimensional single particle tracking at the sub-millisecond scale. Appl. Phys. Lett. 102, 173702 (2013).

46. Ha, T. et al. Single-molecule fluorescence spectroscopy of enzyme conformational dynamics and cleavage mechanism. Proc. Natl Acad. Sci. USA 96, 893-898 (1999).

47. Myong, S., Rasnik, I., Joo, C., Lohman, T. M. \& Ha, T. Repetitive shuttling of a motor protein on DNA. Nature 437, 1321-1325 (2005).

48. Gebhardt, J. C. M. et al. Single-molecule imaging of transcription factor binding to DNA in live mammalian cells. Nat. Methods 10, 421-426 (2013).

49. Ulbrich, M. H. \& Isacoff, E. Y. Subunit counting in membrane-bound proteins. Nat. Methods 4, 319-321 (2007).

50. Cocucci, E., Aguet, F., Boulant, S. \& Kirchhausen, T. The first five seconds in the life of a clathrin-coated pit. Cell 150, 495-507 (2012).

51. Hinde, E., Cardarelli, F., Digman, M. A. \& Gratton, E. In vivo pair correlation analysis of EGFP intranuclear diffusion reveals DNA-dependent molecular flow. Proc. Natl Acad. Sci. USA 107, 16560-16565 (2010).

52. Friedrich, J., Seidel, C., Ebner, R. \& Kunz-Schughart, L. A. Spheroid-based drug screen: considerations and practical approach. Nat. Protoc. 4, 309-324 (2009).

\section{Acknowledgements}

We thank J. Werner for helpful discussion regarding SPT instrumentation, K. Sokolov for providing anti-EGF-biotin complexes, J. Tunnell for the A431 cell line and Z. Gryczynski for the Fluorometer measurements. This work was funded by the Texas 4000, National Institutes of Health (EB011556, NS078791, NS082518 and CA193038), and the American Heart Association (14EIA8970041)

\section{Author contributions}

E.P.P., H.-C.Y. and A.K.D. conceived the project; C.L. performed computational modelling; and E.P.P. and K.H. designed and built the microscope, and developed control software. E.P.P. and Y.-L.L. performed the experiments and analysed the results. Y.-L.L. performed cell culture and sample preparation. C.-K.C. and M.-C.H. helped analyse the EGFR entry pathways. E.P.P., H.-C.Y. and A.K.D. wrote the manuscript. H.-C.Y. and A.K.D. supervised the project.

\section{Additional information}

Supplementary Information accompanies this paper at http://www.nature.com/ naturecommunications

Competing financial interests: The authors declare no competing financial interests.

Reprints and permission information is available online at http://npg.nature.com/ reprintsandpermissions/

How to cite this article: Perillo, E. P. et al. Deep and high-resolution three-dimensional tracking of single particles using nonlinear and multiplexed illumination. Nat. Commun. 6:7874 doi: 10.1038/ncomms8874 (2015).

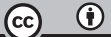

This work is licensed under a Creative Commons Attribution 4.0 International License. The images or other third party material in this article are included in the article's Creative Commons license, unless indicated otherwise in the credit line; if the material is not included under the Creative Commons license, users will need to obtain permission from the license holder to reproduce the material To view a copy of this license, visit http://creativecommons.org/licenses/by/4.0/ 